\title{
Optimal asset allocation using a combination of implied and historical information ${ }^{\star}$
}

\author{
Chi Wan Cheang ${ }^{\mathrm{a}, 1, *}, J_{\text {ose Olmo }}^{\mathrm{b}}$, Tiejun $\mathrm{Ma}^{\mathrm{c}}$, Ming-Chien Sung ${ }^{\mathrm{c}}$, Frank McGroarty ${ }^{\mathrm{c}}$ \\ ${ }^{a}$ Department of Economics, University of Southampton, United Kingdom \\ ${ }^{b}$ Department of Economic Analysis, Universidad de Zaragoza and University of Southampton \\ ${ }^{c}$ Southampton Business School, University of Southampton
}

\begin{abstract}
This paper investigates the contribution of option-implied information for strategic asset allocation for individuals with minimum-variance preferences and portfolios with a variety of assets. We propose a covariance matrix that exploits a mixture of historical and option-implied information. Implied variance measures are proposed for those assets for which option-implied information is available. Historical variance and correlation measures are applied to the remaining assets. The performance of this novel approach for constructing optimal investment portfolios is assessed out-of-sample using statistical and economic measures. An empirical application to a sophisticated portfolio comprised by a combination of equities, fixed income, alternative securities and cash deposits shows that implied variance measures with risk premium correction outperform variance measures constructed from historical data and implied variance without correction. This result is robust across investment portfolios, volatility and portfolio performance metrics, and rebalancing schemes.
\end{abstract}

Keywords: asset allocation, implied volatility, historical volatility, realized variance, Sharpe ratio.

JEL classification: E22, G11, G12, C12.

\footnotetext{
${ }^{\star}$ Parts of the research reported in this paper has been supported by Innovate UK, EPSRC and ESRC as part of a KTP project (10262). We also acknowledge the support from Seven Investment Management and in particular the personal contributions of Christopher Cowell, Alessandro Laurent, Ian Jensen-Humphreys and Christopher Darbyshire. In addition, we are grateful to Johnnie Johnson, Richard McGee and Jason Wang for useful discussions and feedback.

${ }^{*}$ Corresponding author

Email addresses: C.Cheang@soton.ac.uk (Chi Wan Cheang), joseolmo@unizar.es (Jose Olmo), tiejun.ma@soton.ac.uk (Tiejun Ma), M.Sung@soton.ac.uk (Ming-Chien Sung), F.J.Mcgroarty@soton.ac.uk (Frank McGroarty)

${ }^{1}$ Present address: Department of Geography and Planning, University of Liverpool, Roxby Building, 74 Bedford St S, Liverpool, United Kingdom L69 7ZT Email address: C. Cheang@liverpool.ac.uk
} 


\section{Introduction}

The objective of this paper is to examine to what extent option-implied information provides better out-of-sample performance in optimal asset allocation for individuals exhibiting minimum-variance preferences. The empirical evidence in the existing literature supporting option-implied measures of volatility is mixed. Jiang and Tian (2005) and Kostakis et al. (2011), among others, find that there is more information content in optionimplied volatility measures than in historical volatility measures. These authors show this result for a range of popular volatility forecast models. Baker and Wurgler (2006) and Chau et al. (2016) examine the role of option-implied volatility in stock markets and show that investors utilize the information extracted from option-implied measures to inform investment decisions. Other studies evaluate portfolio performance using option-implied information and find that forward-looking volatility measures outperform investment portfolios constructed from historical volatility measures in-sample and out-of-sample, see for instance, Buss and Vilkov (2012), DeMiguel et al. (2013) and Kempf et al. (2014).

The current study aims to investigate further the topic of portfolio performance evaluation using implied information obtained from option prices. We contemplate two related hypotheses on the performance of implied variance/volatility measures. First, we study whether these measures outperform forecasts of the future variance based on historical information. Second, we use these forecasts to compute economic performance measures of different investment portfolios constructed from historical and implied variance measures, and a portfolio that combines both approaches. To the best of our knowledge, the empirical finance literature has not studied yet the suitability of investment portfolios that partially incorporate option-implied information in sophisticated portfolios comprised by very different asset types. This study contributes to the literature by filling this gap. In particular, we propose a conditional covariance matrix that relies on a mixture of optionimplied and historical data. Option-implied information is exploited for those assets that also trade in the derivatives market for options and for which implied variance measures are available. For those assets for which option-implied information is not available we approximate the conditional variance using estimates of the historical variance constructed over rolling windows. The correlation between asset returns in the portfolio is also estimated using historical information. 
The problem of combining information for forecasting the conditional variance-covariance matrix of asset returns is of major relevance for large portfolios comprising different asset types and for which option-implied information is not a viable alternative. We analyze the suitability of our variance-covariance matrix using statistical and economic measures. We consider a portfolio comprised by nine assets including equities, fixed income, alternative securities and cash, varying exposures to countries, sectors, and risk levels. Four of these asset categories representing equities from US, UK, Europe and Japan have option-implied information available. For the remaining asset categories, for example, world corporate bonds and commodity funds, option data are not available. In these cases historical information is the only reliable source of information. We aim to investigate whether it is more informationally efficient to combine option-implied information from equity markets and historical information for the remaining asset classes.

Embedding option-implied information into portfolio optimization problems is not trivial. Standard implied variance (IV) measures extracted from option prices are under risk-neutral expectations. By construction, these IV measures differ from the true (but unknown) future variance which is the variable of interest for portfolio managers. The difference between implied variance and future variance is the variance risk premium (VRP). Hence, in order to use implied variance measures as unbiased estimates of the conditional asset variance the variance risk premium needs to be corrected. To do this, we follow DeMiguel et al. (2013) and conduct an interpolation exercise for obtaining unbiased option-implied variances that incorporate the variance risk premium by filtering out the historical risk premium (up to time $t-1$ ) and keeping only the current period $t$ risk premium embedded in implied volatility measures. By doing so we incorporate investors' perspective of future market uncertainty in our forecasts of the conditional variance.

In the empirical application we construct two estimates of the conditional variance that make use of option-implied information: one is the model-free option-implied variance without risk premium adjustment and the second one is an option-implied measure that incorporates a correction using historical risk premium. To compare the empirical results, we also consider the historical variance of asset returns obtained from rolling windows. We compare the performance of these measures using out-of-sample portfolio performance metrics including portfolio return, volatility, Sharpe ratio and maximum 
drawdown, and apply Diebold and Mariano (1995) predictive ability test to attach statistical significance to the results. We also consider statistical performance measures such as the root mean squared prediction error (RMSPE) and the mean absolute prediction error (MAPE). To assess the robustness of our empirical results, we repeat these exercises to monthly, quarterly and semi-annual rebalancing portfolios. Our empirical results show that option-implied variance, especially the risk-premium-corrected implied variance, exhibits superior out-of-sample forecasting ability than the realized variance measure constructed from historical information. In particular, for a reduced portfolio comprised by equities from the US, UK, Europe and Japan, we find strong empirical evidence suggesting that risk-premium-corrected implied variance outperforms other estimators with regards to economic performance when we consider a global minimum-variance objective function. More importantly, the analysis of sophisticated portfolios comprised by various asset categories for which option data is only available for a subset of them reveals that incorporating risk-premium-corrected implied variance improves portfolio performance. This finding provides strong empirical support to our proposed methodology for forecasting the conditional variance-covariance matrix using a mix of historical and implied information. These results are robust across rebalancing periods.

The remainder of the paper is organised as follows. Section 2 introduces the portfolio optimization problem for our empirical exercise. Section 3 introduces our novel variancecovariance matrix based on a mixture of implied and historical information. Section 4 presents the performance measures. We divide the section into a variance forecasting comparison exercise and a comparison of portfolio performance using standard performance metrics in terms of portfolio return and volatility, Sharpe ratio and maximum drawdown. These comparisons are done in an out-of-sample context. Section 5 provides descriptions of the investment portfolios, portfolio optimization exercise under different portfolio constraints, and a comparison of the forecasting performance for the different conditional variance matrices discussed in the methodology section. Section 6 concludes the study. 


\section{Methodology}

This study examines whether forecasts of the conditional variance of asset returns obtained from forward-looking information in option prices improve the prediction and portfolio performances of sophisticated investment portfolios managed by financial practitioners. We consider an investment portfolio with multiple security segments including equities, fixed-income, cash and other alternative securities. Due to the availability of option data, option-implied variances can only be applied to equity assets. For the remaining asset categories, we estimate the conditional variance of returns and the corresponding correlations using historical data.

Let $R_{t+1}$ be the return on a portfolio defined as

$$
R_{t+1}=\sum_{i=1}^{K} w_{i t} r_{i, t+1},
$$

with $r_{i, t+1}$ the return on the assets comprising the portfolio at time $t+1$ and $w_{i t}$ the portfolio weights denoting the allocation of wealth to the risky assets. Let $w=\left(w_{1 t}, \ldots, w_{K, t}\right)^{\prime}$, the conditional mean portfolio return is $E_{t}\left[R_{t+1}\right]=w_{t}^{\prime} E_{t}\left[r_{t+1}\right]$, with $E_{t}[\cdot]$ denoting the expectation conditional on the information available to the individual at time $t$. The conditional variance of the portfolio is $V_{t}\left[R_{t+1}\right]=w_{t}^{\prime} \Sigma_{t} w_{t}$, where $\Sigma_{t}$ is a $K \times K$ matrix with diagonal elements $\sigma_{i, t}^{2}$ that denote the conditional variance of each asset $r_{i, t+1}$ in the portfolio, and off-diagonal elements $\sigma_{i j, t}$ which are the conditional covariances of assets $i$ and $j$ for all $i \neq j$ and $i, j=1, \ldots K$.

Rational investors prefer a portfolio with high mean return and low variance. The finance literature has formalized this type of preferences using a mean-variance utility function. In this scenario an investor chooses the optimal portfolio weights from maximizing the following objective function

$$
\max _{w_{t}} w_{t}^{\prime} E_{t}\left[r_{t+1}\right]-\frac{1}{2} \gamma w_{t}^{\prime} \Sigma_{t} w_{t}
$$

where $\gamma>0$ denotes the degree of investor's risk aversion.

The linear combination of portfolio mean and variance is maximized subject to a set of equality, inequality and boundary constraints imposed in the portfolio selection. The solution to the mean-variance portfolio requires an estimate of the conditional mean 
return for each asset in the portfolio inducing, in turn, the presence of model risk and measurement error. To account for this, we consider, instead, a global minimum-variance portfolio with optimal weights obtained from minimizing the overall portfolio variance as

$$
\min _{w_{t}} w_{t}^{\prime} \Sigma_{t} w_{t}
$$

subject to the constraints $\sum_{i=1}^{K} w_{i t} r_{i, t+1} \geq \overline{\bar{R}}$, with $\overline{\bar{R}}$ the target return imposed on the portfolio, and $\sum_{i=1}^{K} w_{i t}=1$. We do not allow for short positions on the assets such that the optimal weights are bounded between zero and one, $0 \leq w_{i t} \leq 1$ for $i=1, \ldots, K$.

In the empirical application, we consider an unconstrained version of the minimum variance portfolio and constrained versions given by different values of $\overline{\bar{R}}$ varying from $4 \%$ to $10 \%$. We will also consider a further classification into two types of portfolios: i) an equity portfolio for which implied information from option markets is available for all assets, ii) a sophisticated portfolio that extends the preceding portfolio by considering five more assets without access to implied information.

\subsection{Conditional variance forecasts}

The conditional covariance matrix is an essential input for portfolio optimization. Sample counterparts of the variance and covariance between asset returns provide consistent estimates of the population parameters. We introduce dynamics by estimating the conditional variance using rolling windows. For instance, the conditional covariances at month $t$ are estimated by the sample historical covariances over the last $m$ months as

$$
\widehat{\sigma}_{i j, t}=\frac{1}{m-1} \sum_{s=1}^{m}\left(r_{i, t-s+1}-\bar{r}_{i, t}\right)\left(r_{j, t-s+1}-\bar{r}_{j, t}\right) \quad \text { for all } i, j \in K
$$

where $\bar{r}_{i, t}=\frac{1}{m} \sum_{s=1}^{m} r_{i, t-s+1}$ denotes the sample mean asset return of asset $i$ over the last $m$ months. The structure of rolling windows allows us to drop the oldest observation and incorporate the newest one into the sample for calculating the sample estimate of historical covariances $^{1}$.

\footnotetext{
${ }^{1}$ The above sample estimates assume that the return variation in each period is equally important over the last $m$ months, which is usually not the case. Moving average methods for variance forecasting extend these methods and provide an effective way of estimating the conditional variance over time. In this approach past observations decay logarithmically such that recent data contributes to the sample
} 
An alternative to the use of historical information is to exploit information from option markets. The latter approach introduces investors' forward-looking views on the price formation process. Implied variance from option prices subsumes the historical variance of the underlying asset and provides a more efficient forecast of the future variance. This study focuses on model-free implied variance measures to estimate the future variance of asset returns. These measures do not rely on traditional option valuation models such as Black and Scholes (1973) and, hence, are free of model risk. The model-free approach can be interpreted as a nonparametric estimator of the risk-neutral conditional variance of the stock return until expiration of the stock option. We consider the nonparametric method introduced by the Chicago Board Options Exchange ${ }^{\circledR}$ (CBOE) in 1993 based on the seminal work of Whaley (1993). The CBOE Volatility Index ${ }^{\circledR}$ (VIX) is designed to measure market's expectation of 30-day volatility implied by the out-of-the-money S\&P 500 option prices $^{2}$. The CBOE model-free implied volatility is calculated based on a weighted average of SPX call and put option prices over a wide range of strike prices ${ }^{3}$. The generalised formula for calculating the squared VIX (implied variance) is

$$
\mathrm{VIX}^{2}=100 \times \frac{2}{\bar{T}} \sum_{n} \frac{\Delta K_{n}}{K_{n}^{2}} e^{r_{f}} \bar{T} Q\left(K_{n}\right)-\frac{1}{\bar{T}}\left[\frac{F}{K_{0}}-1\right]^{2}
$$

where $\bar{T}$ is the time to expiration, $F$ is the forward price for the same underlying asset and same maturity, $K_{0}$ is the first strike below the forward price, $K_{n}$ is the strike price of the $n^{\text {th }}$ out-of-money option, $\Delta K_{i}=\frac{K_{n+1}-K_{n-1}}{2}$ is the interval between strike prices on either side of $K_{n}, Q\left(K_{n}\right)$ is the midpoint of the bid-ask spread for each option with strike price $K_{n}$, and $r_{f}$ is the risk-free rate to expiration.

Many studies, see for example, Poon and Granger (2003) and Kostakis et al. (2011), show that the predictions of option-implied variance measures such as equation (5) provide better forecasts than estimators based on historical sample data. Other related studies, see for example, Jorion (1995), Fleming (1998) and Neely (2009) find that implied variance is a biased forecast of future variance and contains incremental information beyond the

\footnotetext{
estimate more than older data, see RiskMetrics technical document by Longerstaey and Spencer (1996) for the exponential moving average (EWMA) effective variance estimate.

${ }^{2}$ The original measure of volatility index is based on at-the-money S\&P100 option prices. CBOE updated the VIX to reflect S\&P500 (SPX) from 2003.

${ }^{3}$ See the CBOE (2009) white paper for the detailed illustration of VIX calculation.
} 
future variance measure. For instance we may have

$$
V R P_{t} \equiv I V_{t}-R V_{t}
$$

where $I V_{t}$ denotes the implied-variance measure and $R V_{t}$ is the realized variance obtained from asset returns at higher frequencies. Realized variance measures are shown to be consistent estimates of the future variance, see Andersen et al. (2003, 2001) and BarndorffNielsen and Shephard (2002). The variance risk premium at time $t$ is the difference between the ex-ante risk-neutral expectation of future variance over the period $t$ to $t+1$ and the expost realized variance computed over the period $t-1$ to $t$. For estimation and forecasting purposes, the measure in (6) is replaced by the following forward-looking measure given by the expected variance risk premium, defined as

$$
E V R P_{t} \equiv I V_{t}-E_{t}\left[R V_{t+1}\right]
$$

The quantity $E_{t}\left[R V_{t+1}\right]$ is computed using time series forecasts. For example, under the assumption that the realized variance is a martingale process, realized variance at time $t$ is the best predictor for realized variance at $t+1$, then equations (6) and (7) are the same.

\section{A novel variance-covariance matrix}

The conditional variance-covariance matrix $\Sigma_{t}$ is the main variable of interest in the portfolio optimization problems in (2) and (3). It is a symmetric positive semi-definite matrix which can be decomposed into a diagonal matrix of conditional variances $D_{t}$ and a matrix of conditional correlations $\Omega_{t}$ such that

$$
\Sigma_{t}=D_{t}^{1 / 2} \Omega_{t} D_{t}^{1 / 2}
$$

We propose to estimate the covariance matrix $\Sigma_{t}$ by exploiting a mixture of implied and historical information on the variability of asset returns in the portfolio. More specifically, under the limitation that information from option prices is only available for a subset of 
the asset categories, we estimate the diagonal matrix $D_{t}$ by the empirical counterpart

$$
\widehat{D}_{t}=\left[\begin{array}{cc}
\widehat{\sigma}_{i v, t}^{2} & 0 \\
0 & \widehat{\sigma}_{h, t}^{2}
\end{array}\right]
$$

with $\widehat{\sigma}_{i v, t}^{2} \equiv I V_{t}$ a $k_{1} \times k_{1}$ diagonal matrix containing the conditional variance of those $k_{1}$ assets for which option-implied information is available. For those assets, we construct option-implied variance measures using the nonparametric model-free measures discussed in the previous section. Similarly, $\widehat{\sigma}_{h, t}^{2}$ denotes a $\left(K-k_{1}\right) \times\left(K-k_{1}\right)$ diagonal matrix that contains the historical variance measures of the remaining $K-k_{1}$ assets in the portfolio. The conditional correlation matrix $\Omega_{t}$ is estimated from historical data. Those measures constructed from historical data are estimated using expression (4) and updated using rolling windows.

The presence of a variance risk premium in option-implied variance measures inflates the overall portfolio variance a shown in (8). Figure B.1 illustrates this stylized fact and shows how the implied variance tends to exceed the historical variance over the entire sample period.

\section{[Insert Figure B.1 about here]}

As mentioned above, we can construct conditional variance estimates obtained from option-implied data by correcting the variance risk premium. We follow the risk premium correction proposed in DeMiguel et al. (2013) and assume that the magnitude of the volatility risk premium is proportional to the level of conditional volatility under the true probability measure. Following these authors, we estimate the monthly historical volatility risk premium, denoted $H V R P_{t}$, as the ratio of monthly average implied volatilities over the corresponding historical volatilities for the past $m$ periods ${ }^{4}$. More formally,

$$
\widehat{\operatorname{HVRP}} t=\frac{\sum_{s=1}^{m} \widehat{\sigma}_{i v, t-s}}{\sum_{s=1}^{m} \widehat{\sigma}_{h, t-s}},
$$

\footnotetext{
${ }^{4}$ DeMiguel et al. (2013) use volatilities instead of variances for correcting the risk premium. The correction for the risk premium term is based on the ratio of the average implied and historical volatilities instead of their difference to avoid potential negative risk premium estimates.
} 
where $\widehat{\sigma}_{i v, t-s}$ is the option-implied volatility of asset $i$ over the past trading dates between $t-m$ and $t-1$, and $\widehat{\sigma}_{h, t-s}$ denotes the estimates of conditional volatility using historical information. Then, assuming that in the next period, from $t$ to $t+1$, the prevailing volatility risk premium can be well approximated by the historical volatility risk premium (10), one can obtain the prediction of the future realized volatility, $\left(R V_{t+1}\right)^{1 / 2}$, which we call the risk-premium-corrected implied volatility and denote as $\widetilde{\sigma}_{i v c, t}$. Hence, the risk-premium-corrected conditional volatility is given by

$$
\widetilde{\sigma}_{i v c, t}=\frac{\widehat{\sigma}_{i v, t}}{\widehat{H V R P}}
$$

We define a new quantity $\widetilde{D}_{t}$ to replace $\widehat{D}_{t}$ in the estimation of variance-covariance matrix $\Sigma_{t}$ in (8). This matrix is defined as

$$
\widetilde{D}_{t}=\left[\begin{array}{cc}
\widetilde{\sigma}_{i v c, t}^{2} & 0 \\
0 & \widehat{\sigma}_{h, t}^{2}
\end{array}\right] .
$$

This matrix only aims to adjust the historical risk premium which does not contain forward-looking information from $t$ to $t+1$. The forward-looking information is still embedded in the risk premium component presented in the option-implied volatility $\hat{\sigma}_{i v, t}$ in (11).

\section{Performance measures}

In this section we analyze different measures of performance that are used to compare the gains of implied information against historical information. We first compare implied variance measures against historical variance measures using statistical loss functions including the RMSPE and MAPE. Next we assess the gains of using implied versus historical information from a portfolio performance perspective in terms of portfolio returns and volatility, Sharpe ratio and maximum drawdown.

\subsection{Variance forecasting performance: implied vs historical}

The inherent problem of conditional variance in a portfolio optimization is that it is unobservable at time $t$. One can calculate realized variance based on high frequency data which asymptotically converges to the integrated variance defined over the low-frequency 
interval of interest. If further assumes that realized variance follows a martingale, the variance at time $t$ is the best predictor for the variance at time $t+1$. Therefore, one might use realized variance to approximate the conditional variance of returns one period ahead (see for instance Andersen et al., 2003 and Bollerslev et al., 2011).

To assess the predictability of the mentioned variance estimators in the previous section we consider realized variance as a benchmark to compare the prediction power. We compute the absolute and relative distance between each of the three mentioned variance estimators and the realized variance obtained from high-frequency data. The expressions of the realized variance, absolute and relative prediction errors for each asset in the portfolio, the aggregate portfolio prediction error, and the prediction criteria (MSPE and MAPE) are explained below.

In our analysis, the monthly realized variance for each stock is constructed based on daily equity prices ${ }^{5}$. The realized variance for a generic asset in a portfolio at month $t$ is

$$
R V_{t}^{(m)}=\sum_{j=1}^{m} r_{t-1+j \Delta}^{2}, \quad t=1, \ldots, T,
$$

where $r_{t-1+j \Delta}$ is the continuously compounded return from time $t-1+(j-1) \Delta$ to $t-1+j \Delta$ in which $\Delta$ is the fraction of a trading session associated with the monthly sampling frequency and $m$ is the number of sampled observations per trading session. For instance, $m$ is number of trading days in a month for the realized variance measure based on daily data.

The absolute prediction error is defined as the difference between the benchmark realized variance which is a proxy of the underlying conditional variance measure and the variance estimate. For the historical variance measure the absolute prediction error is as $e_{h, t} \equiv R V_{t}^{(m)}-\widehat{\sigma}_{h, t}^{2}$, and for the implied variance measure the corresponding prediction error is as $e_{i v, t} \equiv R V_{t}^{(m)}-\widehat{\sigma}_{i v, t}^{2}$. It may be also of interest to measure the prediction error as a fraction of the realized variance. We define these relative measures as $u_{h, t} \equiv \frac{e_{h, t}}{R V_{t}^{(m)}}$ and $u_{i v, t} \equiv \frac{e_{i v, t}}{R V_{t}^{(m)}}$ and capture the relative prediction error with respect to the realized variance measure. As an additional measure of variance forecasting performance we in-

\footnotetext{
${ }^{5}$ Theoretically, the higher frequency improves the accuracy of realized variance estimation. However, due to data availability, daily data is used to estimate the realized variance.
} 
troduce an aggregate portfolio prediction error. This is defined as the sum of the absolute prediction error of each asset $i$ compounded by the square of the corresponding portfolio weight $w_{i}$. More formally, let $v_{t} \equiv \sum_{i=1}^{K} w_{i}^{2} e_{i, t}$ where $e_{i, t}$ denotes the absolute prediction errors computed from different variance forecast measures, and $K$ is the number of assets in the portfolio. For each variance measure we will compute a different aggregate portfolio prediction error.

Using these error measures, suitable loss functions for comparing the forecasting performance of the different variance forecasts are the root mean square prediction error (RMSPE) and the mean absolute prediction error (MAPE). These loss functions are constructed as

$$
\begin{gathered}
\operatorname{RMSPE}=\sqrt{\frac{1}{T} \sum_{t=1}^{T} e_{t}^{2}}, \quad \text { and } \\
\text { MAPE }=\frac{1}{T} \sum_{t=1}^{T}\left|e_{t}\right|,
\end{gathered}
$$

where $e_{t}$ denote the prediction errors of both implied and historical variance forecasts. We also compute the same loss functions for the relative prediction errors $u_{t}$ and the aggregate portfolio error $v_{t}$.

\subsection{Portfolio performance measures}

The performance of the various portfolios obtained under different sets of constraints and choices of variance measures is evaluated using statistical and economic measures. In particular, we choose four criteria which are widely used in the empirical finance literature. These metrics are the 1) portfolio return; 2) portfolio volatility; 3) Sharpe ratio; and 4) maximum drawdown. All these performance metrics are considered in an out-of-sample setting. When implementing the out-of-sample procedure with monthly updates, one needs to form an optimal portfolio on a particular month, say time $t$, and then compute the portfolio return from holding that portfolio for one month, i.e., until time $t+1$. Next, in order to demonstrate the flexibility and robustness of our analysis, portfolios are rebalanced using monthly, quarterly, and semi-annual intervals. All the performance metrics at different rebalancing frequencies are reported at a monthly interval to provide 
meaningful comparisons.

The out-of-sample portfolio return from time $t$ to $t+1$ is computed as

$$
\widehat{R}_{t+1}=w_{t}^{\prime} r_{t+1}
$$

where $w_{t}$ is the vector of optimal weights from the portfolio strategy at time $t$, and $r_{t+1}$ denotes the vector of realization of the asset returns at time $t+1$. In the empirical application we construct the out-of-sample portfolio returns using dynamic portfolio weights that are obtained from the investor's optimal portfolio allocation problem using information computed over rolling windows. For the sake of consistency with the estimation of the historical conditional variances and covariances, we consider rolling windows comprised by $m=60$ months.

\section{[Insert Figure B.2 about here]}

As illustrated by a time-line in figure B.2, the optimal weights are updated every month with the same window length for the entire observation period $T$. The first set of optimal weights is calculated using the sample from $t=1$ to $t=60$ for $t \in T^{6}$. After computing the rolling optimal weights for $T-m-1$ periods, the rolling portfolio returns can be calculated by equation (13) for each period over time $t+1$ to $T$.

The average out-of-sample portfolio return among the entire out-of-sample period is

$$
\bar{R}=\frac{1}{T-m} \sum_{t=m}^{T-1} \widehat{R}_{t+1} .
$$

The out-of-sample portfolio volatility is given by the sample standard deviation of the portfolio returns over the entire out-of-sample period:

$$
\widehat{\sigma}_{R}=\left[\frac{1}{T-m} \sum_{t=m}^{T-1}\left(\widehat{R}_{t+1}-\bar{R}\right)^{2}\right]^{1 / 2}
$$

\footnotetext{
${ }^{6}$ The choice of window size corresponds to five years of observations and conforms with standard practice in the investment industry. The empirical results are not very sensitive, though, to the specific choice of the window size.
} 
such that the out-of-sample portfolio Sharpe ratio (SR) is equal to

$$
\widehat{S R}=\frac{\bar{R}-r_{f}^{m}}{\widehat{\sigma}_{R}}
$$

where $r_{f}^{m}$ denotes the monthly risk-free rate that is assumed to be constant over the out-of-sample evaluation period.

Finally one might be also interested in the measure of portfolio drawdown which is a measurement of decline in portfolio value from a peak to its trough over a period of time. Consider the out-of-sample period from $t=T-m+1$ to $T$, the portfolio drawdown at time $t$ over the out-of-sample period is defined as

$$
D_{t}=\max \left\{0, \max _{u=m+1, \ldots, t-1}\left(\sum_{i=m+1}^{u} \hat{R}_{i}\right)-\sum_{i=m+1}^{t} \hat{R}_{i}\right\}
$$

where $\sum_{i=m+1}^{u} \hat{R}_{i}$ is a sequence of partial sums of the out-of-sample portfolio returns from time $m+1$ to $t$.

\subsubsection{Other rebalancing intervals}

To demonstrate the robustness of the portfolio performance results, we consider other rebalancing intervals that include quarterly and semi-annual holding periods. Typically, for quarterly and semi-annual holding periods, the portfolio return is calculated by multiplying the optimal weights on a particular day by the cumulative returns over the following 3 months and 6 months, respectively. However, this method raises at least two concerns: 1) the performance of a portfolio will depend on the particular starting month chosen for forming the optimal portfolio, and 2) the length of the out-of-sample period for quarterly and semi-annual holding periods is dramatically reduced by three and six times, respectively. This has major implications for the statistical ability of the above performance measures to provide meaningful results.

To address these issues, we adopt DeMiguel et al. (2013)'s rebalancing method to produce a series of overlapping portfolio returns. This method exploits more efficiently the available information set. In this setting, the investor holds the portfolio for $l$ months, for instance, $l=3$ for a quarterly rebalancing period and $l=6$ for semi-annual rebalacing periods. For each month of the out-of-sample period one needs to calculate the monthly 
optimal weight and then hold that portfolio for $l$ months. By doing this, there is a set of overlapping portfolio returns with $T-m-l$ number of out-of-sample observations, where $T$ is the total number of observations and $m$ is the size of rolling horizon. Then, the standardised monthly portfolio return computed from an $l$-month rebalancing scheme is obtained by averaging the overlapping portfolio returns over the out-of-sample period. This quantity is further divided by $l$ in order to obtain a monthly measure. The formal definitions for these quantities follow.

The out-of-sample portfolio return from $t$ to $t+l$ is computed by multiplying the optimal set of weights at time $t$ by the cumulative returns on each asset over $l$ months, as

$$
\widehat{R}_{t+l}^{l}=w_{t}^{\prime} \sum_{\tau=1}^{l} r_{t+\tau},
$$

where $w_{t}$ is the vector of optimal weights from the portfolio strategy at time $t, r_{t+\tau}$ denotes the out-of sample asset returns at time $t+\tau$ for $\tau \leq l \in \mathcal{N}$. For instance, our analysis considers $\tau=1,2,3$ for quarterly and $\tau=1,2, \ldots, 6$ for semi-annual intervals. After collecting the $T-m-l+1$ periods of overlapping portfolio returns, the monthly standardised out-of-sample mean and standard deviation are, respectively,

$$
\begin{aligned}
\bar{R}^{l} & =\frac{1}{T-m-l+1} \sum_{t=m}^{T-l} \widehat{R}_{t+l} \\
\hat{\sigma}_{R}^{l} & =\left[\frac{1}{T-m-l+1} \sum_{t=m}^{T-l}\left(\widehat{R}_{t+l}-\bar{R}^{l}\right)^{2}\right]^{1 / 2} .
\end{aligned}
$$

The monthly portfolio Sharpe ratio $\left(S R^{l}\right)$ for a $l$-month rebalancing scheme is

$$
\widehat{S R}^{l}=\frac{\bar{R}^{l}-r_{f}^{m}}{\widehat{\sigma}_{R}^{l}},
$$

where $r_{f}^{m}$ denotes the monthly risk-free rate. The portfolio drawdown for the $l$-month rebalancing scheme is constructed on the same basis as for the monthly rebalancing scheme introduced above. One only needs to divide each element in the partial sum sequence of portfolio return by $l$ months to calculate the average overlapping returns. Consider the out-of-sample period from $t=m+l$ to $T$, the drawdown for $l$-month rebalancing at time 
$t$ is given by

$$
D_{t}=\max \left\{0, \max _{u=m+1, \ldots, t-1}\left(\frac{1}{l} \sum_{i=m+l}^{u} \hat{R}_{i}^{l}\right)-\frac{1}{l} \sum_{i=m+l}^{t} \hat{R}_{i}^{l}\right\}
$$

where $\frac{1}{l} \sum_{i=m+l}^{u} \hat{R}_{i}^{l}$ is a sequence of partial sums of the out-of-sample overlapping portfolio returns averaged by $l$ months from time $m+l$ to $t$.

\section{Empirical Analysis}

The empirical application mimics a realistic investment portfolio managed by financial practitioners. The portfolio description and implied variance data collection are described in Section 5.1. The three different methods to calculate portfolio variances based on option data and historical data discussed in previous sections are considered now as inputs in the portfolio optimization problem. In Section 5.2, we compare the predictive performance of these variance measures using RMSPE and MAPE measures. In Section 5.3, we carry out an out-of-sample portfolio performance comparison for different types of portfolios in terms of asset composition, portfolio constraints and rebalancing schemes.

\subsection{Data}

Our data consists of nine asset categories in four different market segments, including equities, fixed income, alternative securities and cash, varying exposures to countries, sectors and risk levels. We aim to derive an easily implementable portfolio for small scale investors and we do not consider currency hedging ${ }^{7}$. To convert index levels from different currencies into one common currency, we use Pound Sterling (GBP) denominated indices extracted from Bloomberg. The list of assets in the proposed portfolio is found in table A.1.

\section{[ Insert Table A.1 about here ]}

When implementing portfolio optimizations we use monthly log-returns to compute the monthly rebalancing portfolios. The dataset covers the period from January 2001 to

\footnotetext{
${ }^{7}$ For professional or large scale practitioners, it is reasonable to manage the currency risk by using currency futures or investing in currency markets.
} 
December 2017, with a total of 204 monthly observations available. The first estimation window for the estimates of covariance matrices is from January 2001 to December 2005, with a window size of 60 months $^{8}$. The out-of-sample period is from January 2006 to December 2017. For every month in the out-of-sample period, the optimal weights are recalculated by obtaining the rolling estimates of the covariance matrices. There are 144 out-of-sample months for the comparison of portfolio performances. The option-implied volatility index captures the annualised risk-neutral implied volatility (standard deviation) in percentage terms, thus the implied variance series $I V_{t}$ is obtained by the square root of implied volatility index. When calculating the monthly implied variance $\left(I V_{t}^{m}\right)$, one needs to transform the value of implied variance by

$$
I V_{t}^{m}=\frac{1}{12}\left(\frac{I V_{t}}{100^{2}}\right)
$$

\subsection{Variance forecasting performance}

As a preliminary exercise we investigate the forecasting performance of the historical variance, risk-premium-corrected implied variance and the unadjusted implied variance among four equity markets, including US S\&P 500, UK FTSE 100, European STOXX 50, and Japanese NIKKEI 225. The variance forecasting performance is carried out in terms of absolute, relative and aggregate prediction errors and assessed by computing the RMSPE and MAPE loss functions. The results are presented in table A.2. The magnitude of the loss functions is very small for all of the variance predictors individually, which suggests that all of them forecast realized variance reasonably well. Nevertheless, there is clear evidence of an outperformance of implied variance measures (with and without risk premium adjustment) as the values of the RMSPE and MAPE are smaller than for the historical variance estimates. We also look at the relative prediction criteria in order to compare if the magnitudes of the prediction errors are relatively sizeable among the different variance estimators.

[ Insert Table A.2 about here ]

\footnotetext{
${ }^{8}$ Option-implied data on the selected assets are only available from Bloomberg from January 2001. This fact conditions the start date of our portfolio that contains both stock prices and options data. Our choice of rolling window is driven by standard practices in the financial industry.
} 
Panel A in table A.2 presents mixed evidence. The raw implied variance measures outperform the other forecasting methods for the MAPE in most cases. For the Nikkei index, though, the risk-premium-corrected implied variance is the best performer. The results in Panel B are more robust. In this case we find that the risk-premium-corrected implied variance has smallest RMSPE among all the equity markets. Moreover, the value of these loss functions for the aggregate prediction errors demonstrates that aggregation of errors across assets in the portfolio does not cancel out the prediction error of each other. In this case the implied variance still outperforms the historical variance in terms of aggregate forecasting performance. This suggests that the improvement in forecasting ability of the implied variance measures is robust across assets with implied information.

\subsection{Out-of-sample portfolio performance}

The analysis of portfolio performance is divided into two parts. First, our discussion starts with an equity portfolio which contains only four equity indices in UK, US, Europe and Japan. It aims to examine the direct effect of using implied variance for equity portfolios, without mixing (diluting/amplifying) the effect from historical information. Second, to assess this effect on a portfolio that also contains other asset categories for which there is no derivatives market, we also entertain the full portfolio comprised by nine assets. The optimal portfolio allocation in both portfolios is obtained by minimizing the portfolio variance. For each portfolio, we solve two different optimal portfolio allocation problems. First, we entertain a minimum-variance portfolio that is determined by a target expected portfolio return. In order to assess the sensitivity of the results to the choice of the target return in the portfolio allocation problem, we repeat the optimization exercise for values of the target portfolio return given by $4 \%, 6 \%, 8 \%$ and $10 \%$. We also include a cash liquidity restriction that ranges within $5 \%-20 \%$, that is, we impose that the minimum cash holdings in the optimal portfolio are in this range. As an additional exercise, we also obtain the optimal portfolio allocation of the unconstrained minimum variance portfolio exercise. Finally, for each portfolio, we derive the optimal portfolio weights using three different types of conditional covariance matrices $\Sigma_{t}$ in the investor's objective function (2). One of these matrices is constructed only using historical data and the other two are based on a mix of implied and historical information.

In summary, optimization results are reported in terms of two different asset compo- 
nents (equity-only vs. full assets), for a constrained and unconstrained minimum-variance objective function and using three different methods for forecasting the conditional variance. Lastly, as a robustness exercise, we repeat the empirical analysis for quarterly and semi-annual rebalancing intervals.

\section{Performance of the equity portfolio}

The average monthly out-of-sample performance metrics over 2006 to 2017 for the equity investment portfolio comprised by the four financial indices are reported in Panel A of table A.3, where the best performance metrics for among different variance estimates are indicated in bold style. We apply Diebold and Mariano (1995) tests to assess statistically the significance of our results. In particular, we report two sets of $p$-values in parenthesis. The top $p$-values correspond to the null hypothesis that the portfolio performance is not better than those calculated using the realized variance. The bottom $p$-values correspond to the null hypothesis that the portfolio performance is not better than for the method using the historical variance. In both cases a small $p$-value suggests rejecting the null hypothesis that the portfolio being evaluated is not better than the benchmark ${ }^{9}$.

The optimal weights obtained from the different methods to forecast the variance and under different sets of portfolio constraints ${ }^{10}$ are illustrated in figure B.3. On average, over the entire out-of-sample period, the use of implied variance (with and without risk premium correction) provides better portfolio returns and Sharpe ratio with at least $10 \%$ significant $p$-values. In contrast, the optimal portfolio weights obtained under the historical variance approach yield investment portfolios with lower out-of-sample volatility. The outof-sample maximum drawdown metric provides mixed results across portfolios.

\section{[ Insert Panel A of Table A.3, Figure B.3 about here ]}

\section{Performance of the full portfolio}

The average out-of-sample performance metrics for the entire investment portfolio are reported in Panel B of table A.3. The optimal weights obtained from the different variance

\footnotetext{
${ }^{9}$ We acknowledge that these $p$-values could be improved by providing a single $p$-value obtained from a test comparing multiple forecasting models, see White (2000) and Hansen (2005). However, by doing so we would not be able to obtain evidence of the pairwise superior predictive ability across forecasting methods, which is the object of interest in this paper.

${ }^{10}$ For illustration purpose, we only report the figures of the optimal weights for the unconstrained global minimum-variance portfolio and the constrained version of the portfolio obtained imposing a target portfolio return of $4 \%$.
} 
estimates under different sets of portfolio constraints ${ }^{11}$ are illustrated in figure B.4. The implied variance with risk premium correction outperforms other variance estimates across the different performance metrics: portfolio return, volatility and Sharpe ratio, although the gains are not as sizeable as for the equity-only portfolio. This can be observed in the statistical significance of Diebold and Mariano (1995) $p$-values. This is an interesting observation that highlights the effect of mixing implied variance and historical information in the covariance matrix of the full portfolio. As we increase the number of assets in the portfolio without implied information we need to rely more on historical information. Our results suggest that this circumstance has a negative effect on the overall statistical and economic portfolio performance.

\section{[ Insert Panel B of Table A.3, Figure B.4 about here ]}

\section{Portfolio performance for other rebalancing intervals}

In tables A.4 and A.5 we report the performance of both the equity and full portfolios for quarterly and semi-annual rebalancing intervals. The improvement in portfolio performance obtained by using implied variance with risk premium correction is remarkable and robust across different rebalancing frequencies. In addition, it is noticeable that all the variance estimators exhibit larger portfolio variance than under the one-month rebalancing scheme.

\section{[ Insert Tables A.4 and A.5 about here ]}

\section{Conclusion}

We analyze the forecasting and portfolio performance of option-implied variance measures in sophisticated portfolios comprised by different asset categories involving equity, corporate bonds, commodity funds and cash. The advantages of using option-implied information in portfolio optimization have been examined in literature. However, in this paper we introduce a novel strategy to forecast the variance-covariance matrix when there is no option-implied information on all of the assets in the portfolio. We propose a variancecovariance matrix that combines forward-looking information from option markets with

\footnotetext{
${ }^{11}$ See footnote 10 .
} 
historical information on the asset returns. More specifically, we forecast the conditional variance of those assets for which there are option prices using model-free estimates of the implied variance. For those assets for which this information is not available we rely on estimates of the conditional variance using historical data computed over rolling windows.

In the empirical analysis for two investment portfolios characterized by different sets of constraints, we find that option-implied variance, especially the risk-premium-corrected one, exhibits superior forecasting ability than historical measures of the conditional variance. These results are confirmed for an analysis of portfolio performance out-of-sample. We find strong empirical evidence suggesting that the risk-premium-corrected implied variance outperforms other estimators among most of the scenarios characterized by different portfolio constraints and rebalancing frequencies. We also find that for the equity only scenario, the implied variance with variance-risk-premium correction consistently improves the other investment portfolios with regards to the portfolio returns and Sharpe ratio.

We assess these gains in portfolio performance for a sophisticated portfolio that not only contains equities but also other asset categories such as corporate bonds and commodity funds for which there is no options data. In this case we replace the fully implied variance approach by the mixed approach that combines implied variance with historical variance. Our results also confirm the outperformance of optimal portfolio allocations obtained from the mixed approach compared to simply using historical information. In particular, we find superior out-of-sample portfolio returns, portfolio volatility and Sharpe ratio for the covariance matrix that exploits estimates of the implied variance measure using a variance risk premium correction. 


\section{References}

Andersen, T. G., Bollerslev, T., Diebold, F. X., and Labys, P. (2001). The distribution of realized exchange rate volatility. Journal of the American statistical association, 96(453):42-55.

Andersen, T. G., Bollerslev, T., Diebold, F. X., and Labys, P. (2003). Modeling and forecasting realized volatility. Econometrica, 71(2):579-625.

Baker, M. and Wurgler, J. (2006). Investor sentiment and the cross-section of stock returns. The journal of Finance, 61(4):1645-1680.

Barndorff-Nielsen, O. E. and Shephard, N. (2002). Econometric analysis of realized volatility and its use in estimating stochastic volatility models. Journal of the Royal Statistical Society: Series B (Statistical Methodology), 64(2):253-280.

Black, F. and Scholes, M. (1973). The pricing of options and corporate liabilities. Journal of political economy, 81(3):637-654.

Bollerslev, T., Gibson, M., and Zhou, H. (2011). Dynamic estimation of volatility risk premia and investor risk aversion from option-implied and realized volatilities. Journal of econometrics, 160(1):235-245.

Buss, A. and Vilkov, G. (2012). Measuring equity risk with option-implied correlations. Review of Financial Studies, 25(10):3113-3140.

CBOE, C. B. O. E. (2009). The cboe volatility index-vix. White Paper, pages 1-23.

Chau, F., Deesomsak, R., and Koutmos, D. (2016). Does investor sentiment really matter? International Review of Financial Analysis, 48:221-232.

DeMiguel, V., Plyakha, Y., Uppal, R., and Vilkov, G. (2013). Improving portfolio selection using option-implied volatility and skewness. Journal of Financial and Quantitative Analysis, 48(6):1813-1845.

Diebold, F. X. and Mariano, R. S. (1995). Comparing predictive accuracy. Journal of Business and Economic Statistics, 13(3):253-263.

Fleming, J. (1998). The quality of market volatility forecasts implied by s\&p 100 index option prices. Journal of empirical finance, 5(4):317-345.

Hansen, P. R. (2005). A test for superior predictive ability. Journal of Business 8 Economic Statistics, 23(4):365-380.

Jiang, G. J. and Tian, Y. S. (2005). The model-free implied volatility and its information content. Review of Financial Studies, 18(4):1305-1342.

Jorion, P. (1995). Predicting volatility in the foreign exchange market. The Journal of Finance, 50(2):507-528.

Kempf, A., Korn, O., and Saßning, S. (2014). Portfolio optimization using forward-looking information. Review of Finance, 19(1):467-490.

Kostakis, A., Panigirtzoglou, N., and Skiadopoulos, G. (2011). Market timing with optionimplied distributions: A forward-looking approach. Management Science, 57(7):12311249 . 
Longerstaey, J. and Spencer, M. (1996). Riskmetricstm-technical document. Morgan Guaranty Trust Company of New York: New York, 51:54.

Neely, C. J. (2009). Forecasting foreign exchange volatility: Why is implied volatility biased and inefficient? and does it matter? Journal of International Financial Markets, Institutions and Money, 19(1):188-205.

Poon, S.-H. and Granger, C. W. (2003). Forecasting volatility in financial markets: A review. Journal of economic literature, 41(2):478-539.

Whaley, R. E. (1993). Derivatives on market volatility: Hedging tools long overdue. The journal of Derivatives, 1(1):71-84.

White, H. (2000). A reality check for data snooping. Econometrica, 68:1097-1126. 


\section{Appendix A. Tables}

Table A.1: The composition of assets in the portfolios.

\begin{tabular}{llll}
\hline Asset category & Asset & Benchmark & Local currency \\
\hline Equities & US Equity & S\&P 500 & USD \\
& European Equity & STOXX 50 & EUR \\
& UK Equity & FTSE 100 & GBP \\
& Japanese Equity & NIKKEI 225 & multi-currency \\
\hline Fixed Income & Government Bonds & FTSE Russell World Government Bond Index & USD \\
& Corporate Bonds & FTSE Russell US Broad Investment-Grade Bond Index & USD \\
\hline Alternatives & Commodities & S\&P Commodity Index (GSCI) & USD \\
\hline Cash & Cash & LIBOR GBP 3 Month & GBP \\
\hline
\end{tabular}

Table 1 summarises the composition of assets in the portfolios. We consider a sophisticated portfolio which contains nine assets in four different sectors, including equities, fixed income, alternative securities and cash. The selection of assets is diversified across different regions, sectors and risk levels. The end-of-month prices of each benchmark asset is collected from Bloomberg database terminal. To convert the indices at different local currencies to one single currency, we consider the GBP-denominated prices. The sample period covers 204 months from January 2001 to December 2017. In the empirical analysis, the equity-only portfolio considers four equity assets in the equity category; while the full-asset portfolio considers all the assets stated above. 
Table A.2: Variance forecasting performance in terms of RMSPE and MAPE loss functions.

Panel A. Absolute Prediction Error Criteria $\varepsilon_{t}$.

\begin{tabular}{llccc}
\hline & & Historical variance & Implied variance (adj.) & Implied variance \\
\hline S\&P500 & RMSPE & 0.0068 & 0.0065 & $\mathbf{0 . 0 0 4 8}$ \\
& MAPE & 0.0026 & 0.0024 & $\mathbf{0 . 0 0 2 2}$ \\
STOXX50 & RMSPE & 0.0064 & 0.0059 & $\mathbf{0 . 0 0 4 9}$ \\
& MAPE & 0.0030 & 0.0028 & $\mathbf{0 . 0 0 2 8}$ \\
FTSE100 & RMSPE & 0.0055 & 0.0053 & $\mathbf{0 . 0 0 4 2}$ \\
& MAPE & 0.0022 & 0.0020 & $\mathbf{0 . 0 0 2 0}$ \\
NIKKEI225 & RMSPE & 0.0094 & $\mathbf{0 . 0 0 9 0}$ & 0.0093 \\
& MAPE & 0.0033 & $\mathbf{0 . 0 0 3 2}$ & 0.0038 \\
\hline
\end{tabular}

Panel B. Relative Prediction Error Criteria $u_{t}$.

\begin{tabular}{llccc}
\hline & & Historical variance & Implied variance (adj.) & Implied variance \\
\hline S\&P500 & RMSPE & 1.6991 & $\mathbf{1 . 0 9 1 1}$ & 1.9520 \\
& MAPE & 1.1912 & $\mathbf{0 . 7 5 8 1}$ & 1.3549 \\
\multirow{2}{*}{ STOXX50 } & RMSPE & 1.3989 & $\mathbf{0 . 7 6 0 8}$ & 1.2993 \\
& MAPE & 0.8518 & $\mathbf{0 . 6 0 1 0}$ & 0.9529 \\
FTSE100 & RMSPE & 1.7130 & $\mathbf{0 . 8 0 0 9}$ & 1.4932 \\
& MAPE & 0.8688 & $\mathbf{0 . 5 9 6 8}$ & 1.0399 \\
NIKKEI225 & RMSPE & 1.1311 & $\mathbf{0 . 9 5 7 6}$ & 1.3176 \\
& MAPE & 0.7677 & $\mathbf{0 . 6 2 0 7}$ & 0.9571 \\
\hline
\end{tabular}

Panel C. Aggregate Prediction Error Criteria $v_{t}$.

\begin{tabular}{llccc}
\hline & & Historical variance & Implied variance (adj.) & Implied variance \\
\hline \multirow{2}{*}{ Aggregate } & RMSPE & 0.0067 & 0.0063 & $\mathbf{0 . 0 0 4 8}$ \\
& MAPE & 0.0025 & $\mathbf{0 . 0 0 2 3}$ & 0.0023 \\
\hline
\end{tabular}

Table 2 compares the forecasting performance by different variance estimators in predicting realized variance. Since the sample period between January 2001 and December 2005 is the first rolling window (6o months) in the historical variance, the average prediction performance for each variance estimate are analyzed from January 2006 to December 2017. The numbers in bold style illustrate the best forecasting performance. Panel A and B of table 2 respectively reports the absolute and relative prediction errors among the historical variance, implied variance with risk premium correction and implied variance corresponding to individual equity markets. Panel $\mathrm{C}$ of table 2 reports the aggregate prediction error of each variance estimate at the portfolio level, by taking the weights of the corresponding equity assets into account. This aggregate prediction result is based on the optimal weights obtained from the global minimum-variance equity portfolio under different variance estimates. 


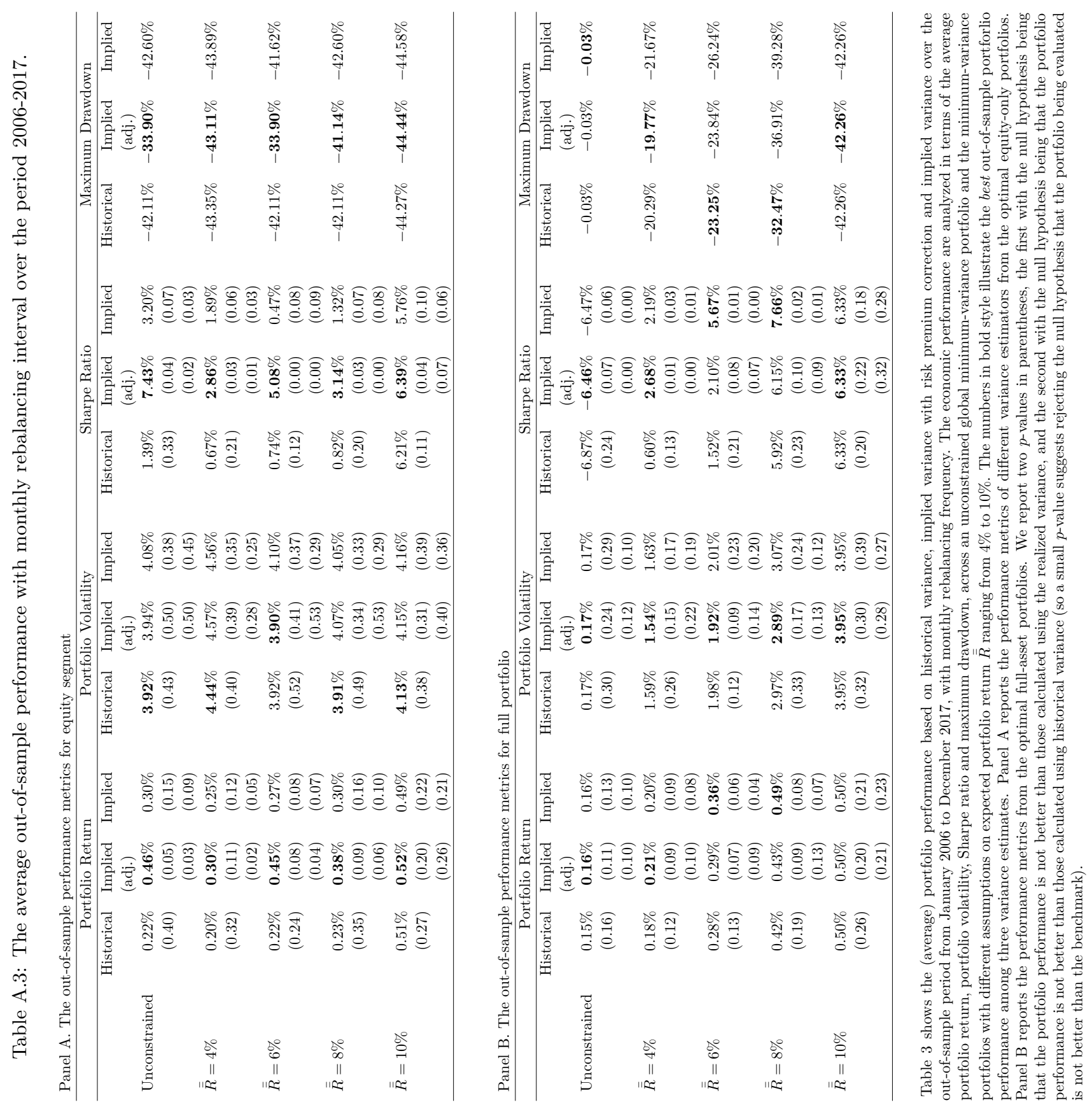




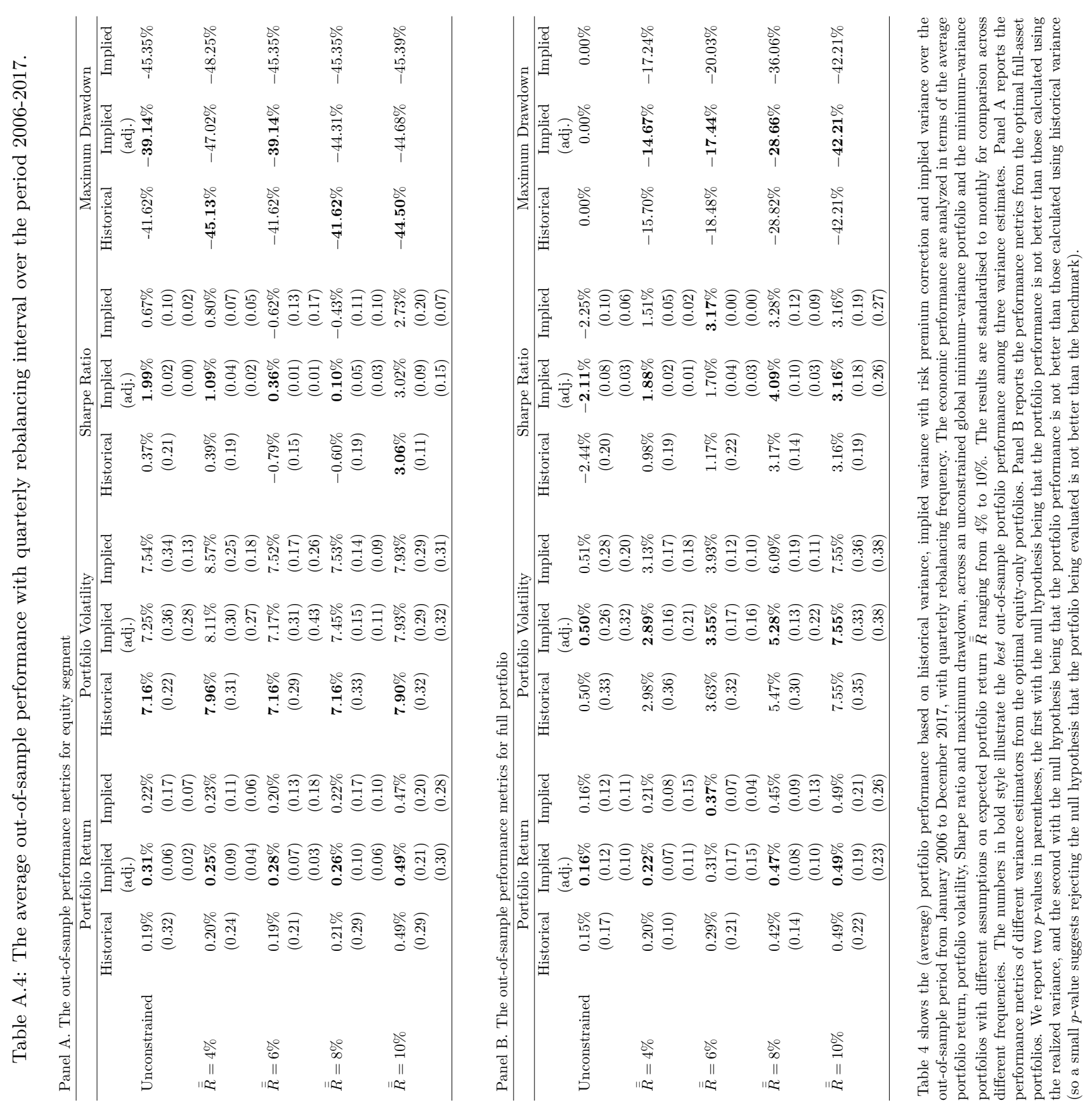




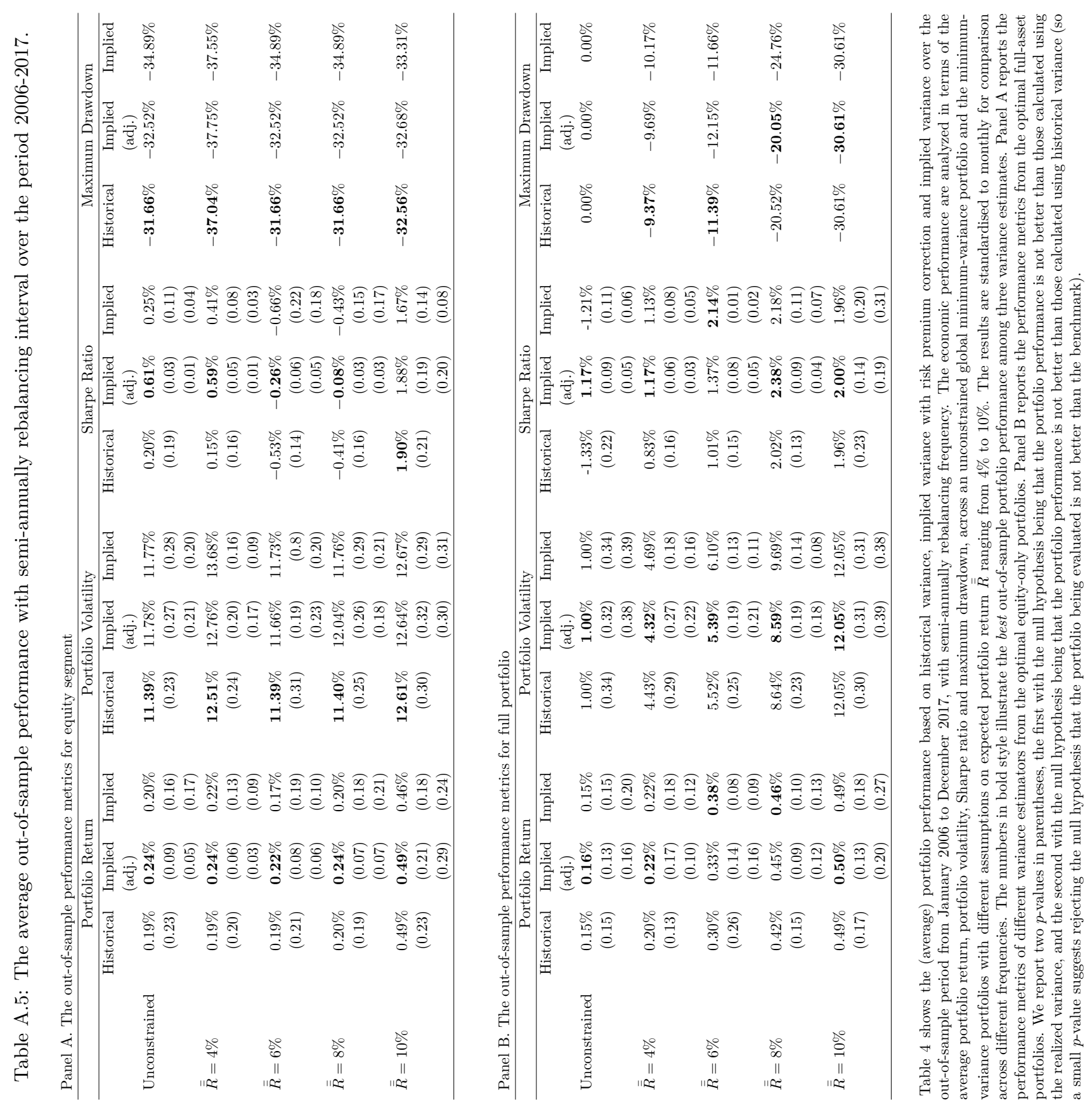




\section{Appendix B. Figures}

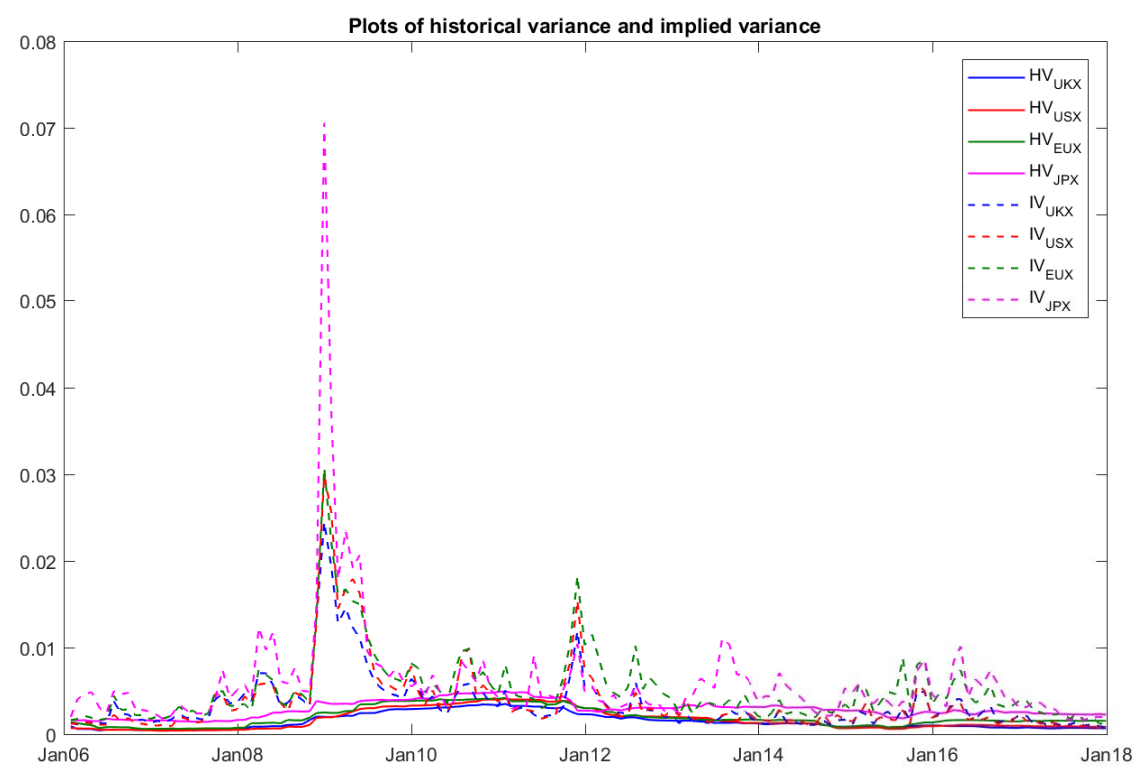

Figure B.1: Plots of monthly historical variances (the solid lines) against implied variances (the dotted lines) over January 2006 - December 2017. The historical variance at each period is calculated by rolling window with a sample size of 60 months. The implied variance is calculated by converting the (annualised) model-free implied volatility index into monthly value as monthly implied variance $=\frac{1}{12}\left(\frac{\text { implied volatility }}{100}\right)^{2}$.

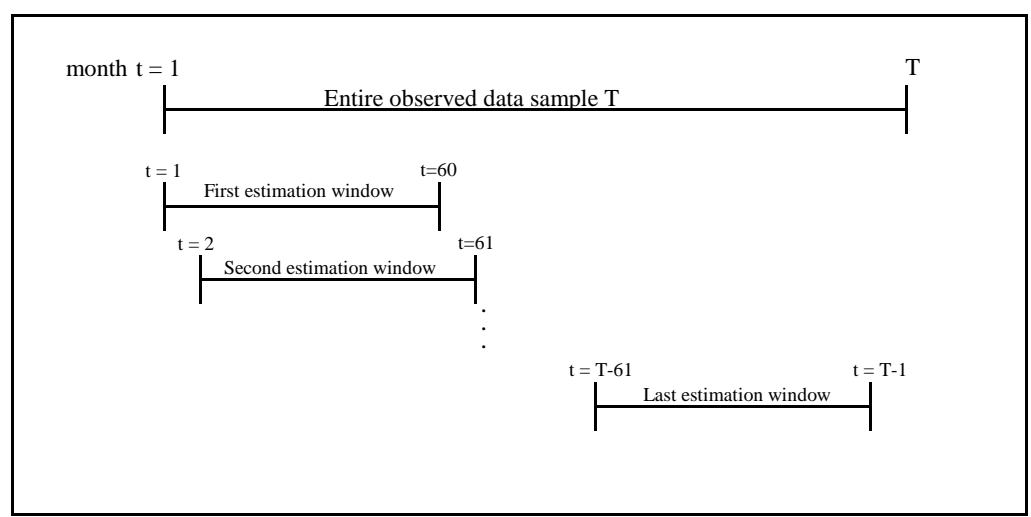

Figure B.2: The out-of-sample rolling horizons with a window size of 60 months. In our empirical exercise, denote $T=204$ as the entire observation period from January 2001 to December 2017. Assume that the width of window is $m=60$ months ( 5 years), the optimal weights are calculated from $t=60$ to $t=203$, there are 144 sets of optimal weights over the sample period. Hence, the out-of-sample portfolio metrics (returns, volatility, Sharpe ratio and drawdown) can be calculated over time $t=61$ to $t=T=204$. 


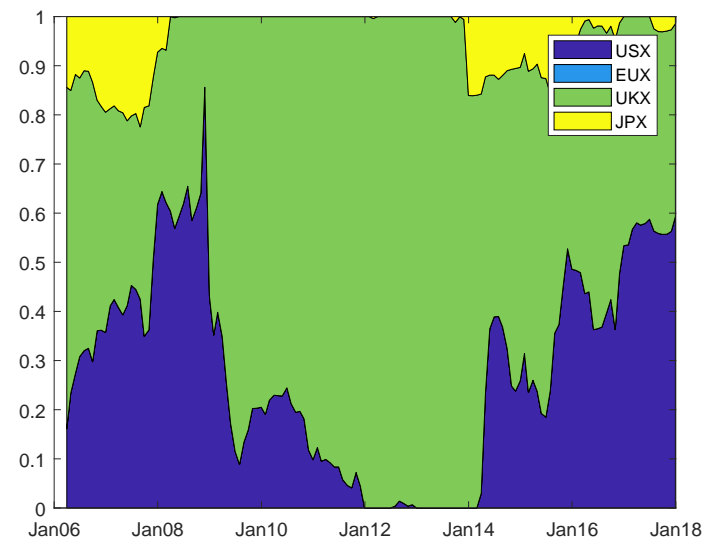

(a) Historical: unconstrained

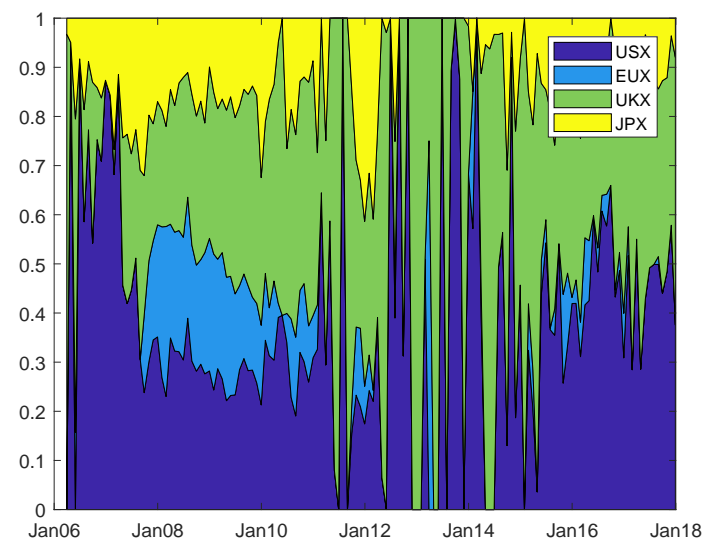

(c) Implied with VRP correction: unconstrained

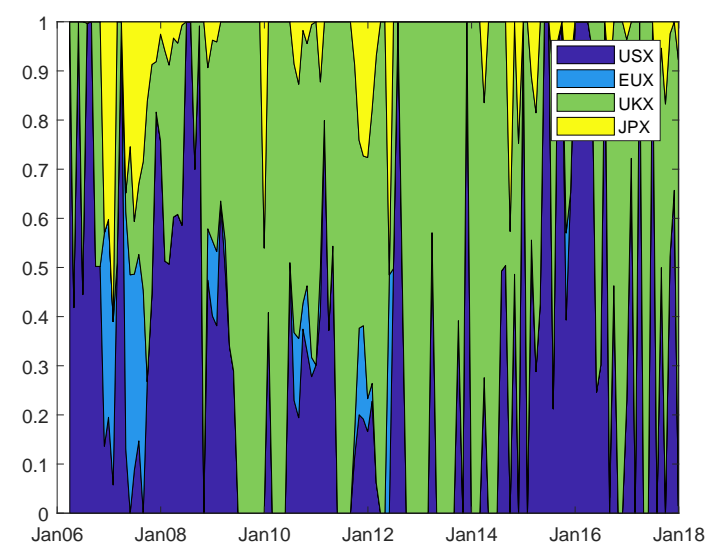

(e) Implied: unconstrained

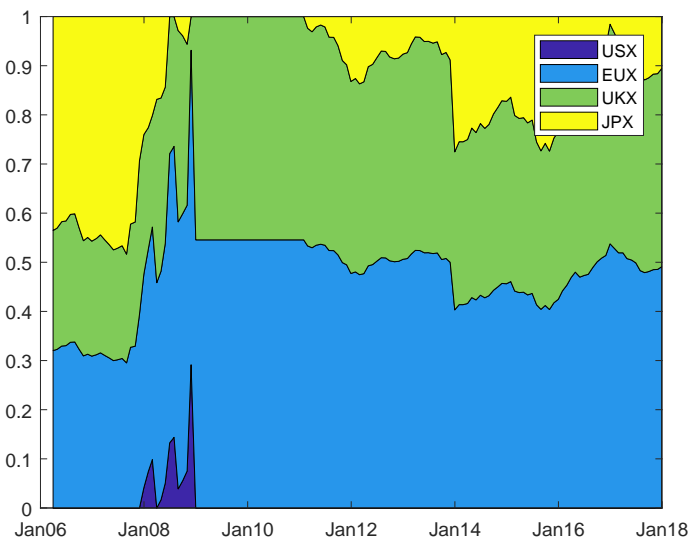

(b) Historical: target return $=4 \%$

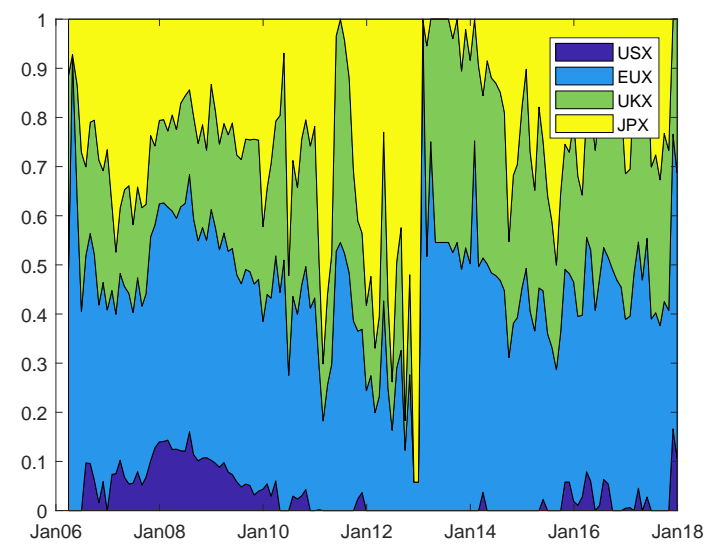

(d) Implied with VRP correction: target return $=4 \%$

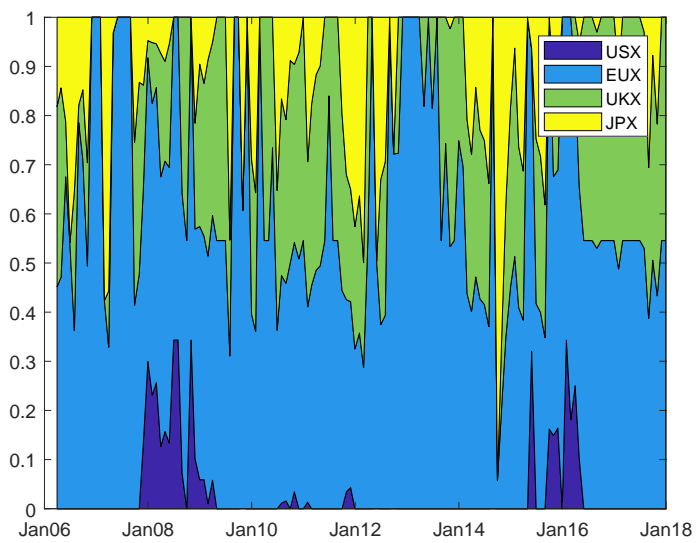

(f) Implied: target return $=4 \%$

Figure B.3: Rolling optimal weights based on different variance estimators for equity portfolios under minimum-variance preference. Figure 3(a), (c), (e) illustrate the optimal weights from equity portfolio based on historical variance, implied variance with VRP correction and implied variance, respectively. Figure 3(b), (d), (f) illustrate the optimal weights from equity portfolio with $4 \%$ expected portfolio return based on historical variance, implied variance with VRP correction and implied variance, respectively. The dynamics of optimal weights over time based on historical variance seem to be less fuzzy. 


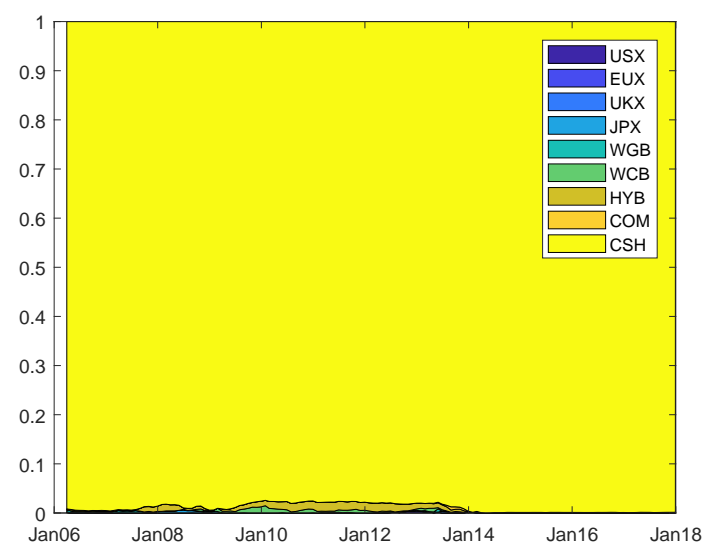

(a) Historical: unconstrained

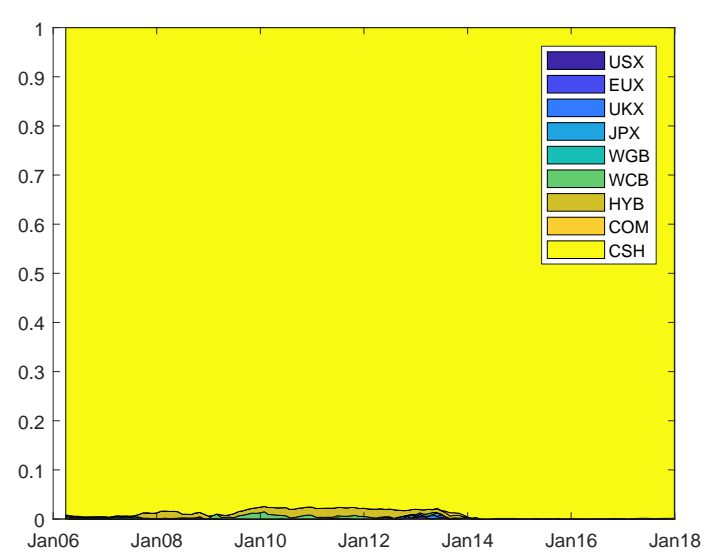

(c) Implied with VRP correction: unconstrained

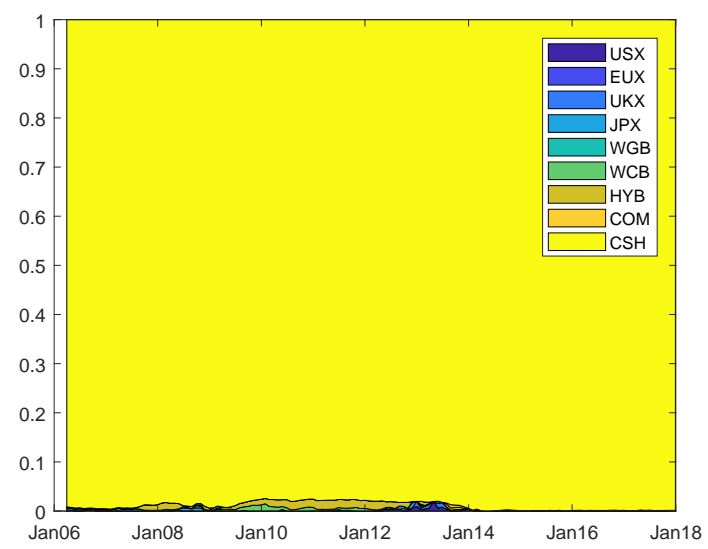

(e) Implied: unconstrained

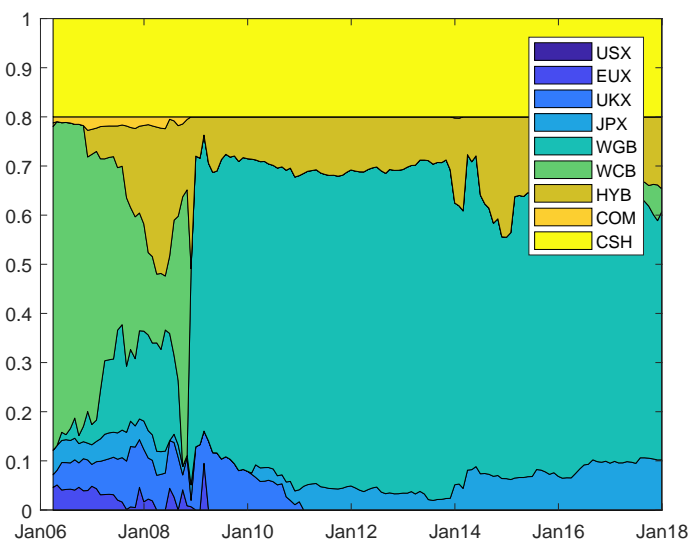

(b) Historical: return $=4 \%$ and cash liquidity $5-20 \%$

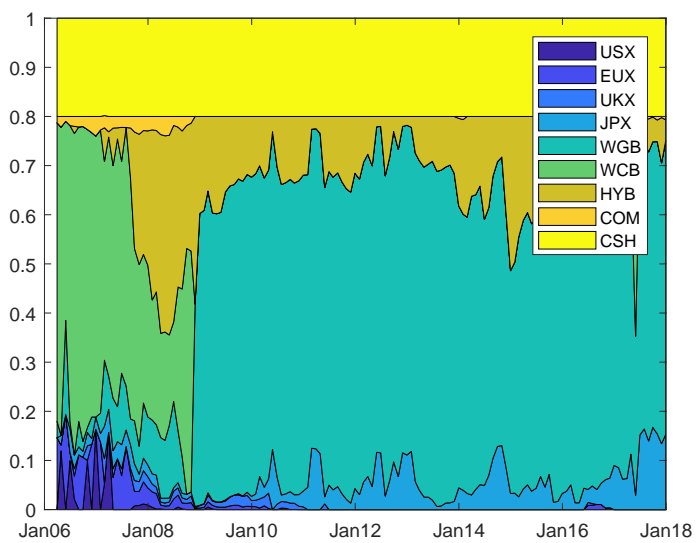

(d) Implied with VRP correction: return $=4 \%$ and cash liquidity $5-20 \%$

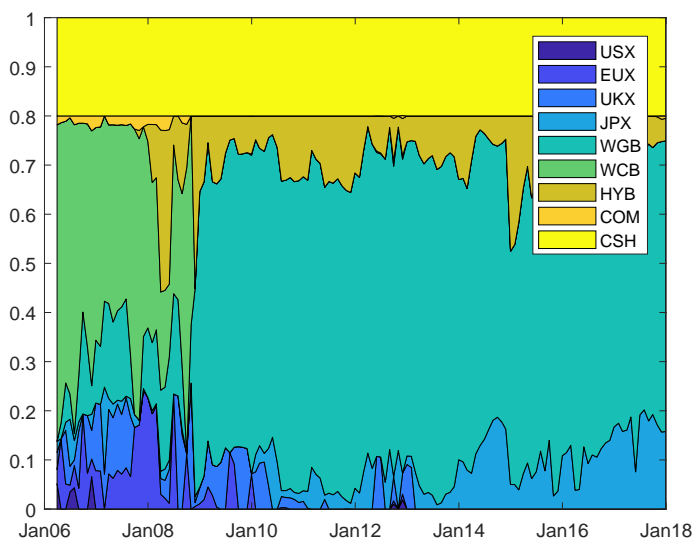

(f) Implied: return $=4 \%$ and cash liquidity $5-20 \%$

Figure B.4: Rolling optimal weights based on different variance estimators for full-asset portfolio under minimum-variance preference. Figure 4(a), (c), (e) illustrate the optimal weights from an unconstrained full-asset portfolio based on historical variance, implied variance with VRP correction and implied variance, respectively. Figure 4(b), (d), (f) illustrate the optimal weights from full-asset portfolio with $4 \%$ expected portfolio return and cash liquidity at 5-20\% based on historical variance, implied variance with VRP correction and implied variance, respectively. Based on the minimum-variance strategy in the unconstrained portfolio, all the variance estimators consistently suggest allocating wealth to cash deposit in order to minimize portfolio risk. By imposing a limited amount of cash liquidity (5-20\%) and an expected portfolio return at $4 \%$, a significant amount of wealth is reallocated to other asset categories such as bonds and equities. 